\title{
Impact of direct-to-consumer advertising for hereditary breast cancer testing on genetic services at a managed care organization: A naturally-occurring experiment
}

Judy Mouchawar, MD, MSPH ${ }^{1}$, Sharon Hensley-Alford, $M P H^{2}$, Suzanne Laurion, $P h D^{3}$, Jennifer Ellis, MSPH ${ }^{1}$, Alanna Kulchak-Rahm, MS ${ }^{1}$, Melissa L. Finucane, $\mathrm{PhD}^{4}$, Richard Meenan, $\mathrm{PhD}, \mathrm{MPH}^{5}$, Lisen Axell, MS $\mathrm{S}^{6}$, Rebecca Pollack, $M S^{2}$, and Debra Ritzwoller, $P h D^{1}$

\begin{abstract}
Purpose: To describe the impact of Myriad Genetics, Inc.'s direct-to-consumer advertising (DTC-ad) campaign on cancer genetic services within two Managed Care Organizations, Kaiser Permanente Colorado (KPCO), Denver, Colorado, where the ad campaign occurred, and Henry Ford Health System (HFHS), Detroit, Michigan, where there were no advertisements. Methods: The main outcome measures were the changes in number and pretest mutation probability of referrals approved for cancer genetic services at KPCO and HFHS during the campaign versus the year prior, and mutation probability of those undergoing testing. Results: At KPCO, referrals increased $244 \%$ during the DTC-ad compared to the same time period a year earlier $(P$ value $<0.001)$. The proportion of referrals at high pretest probability of a mutation ( $10 \%$ or greater) dropped from $69 \%$ the previous year to $48 \%$ during the campaign $(P$ value $<0.001)$. There was no significant change in pretest mutation probability among women who underwent testing between the two time periods. HFHS reported no significant change between the two time periods for numbers or mutation probability of referrals, or for mutation probability of women tested. Conclusion: The DTC-ad caused significant increase in demand for cancer genetic services. In the face of potential future DTC-ad for inherited cancer risk, providers and payers need to consider the delivery of genetic services and genetic education for persons of all risk levels. Genet Med 2005:7(3):191-197.
\end{abstract}

Key Words: direct-to-consumer, advertising, genetics, breast cancer, BRCA1

In the mid-1990s germline mutations in the BRCA1 and BRCA2 (BRCA1/2) genes were found to be associated with an increased risk for breast and ovarian cancers. ${ }^{1,2}$ Direct sequencing for these two genes is currently under patent with Myriad Genetic Laboratories, Inc (Myriad), a biotechnical laboratory in Salt Lake City, Utah. ${ }^{3}$

In May 2002, the biotechnical laboratory began educational outreach to providers in the Denver and Atlanta Metropolitan Areas to prepare them for a direct-to-consumer advertising campaign (DTC-ad) for genetic testing for inherited mutations in the BRCA1/2 genes ("BRACAnalysis"). In preparation for the DTCad, Kaiser Permanente Colorado (KPCO) instituted extensive provider education and set up a patient telephone informational

\footnotetext{
From ${ }^{1}$ Kaiser Permanente Clinical Research Unit, Denver, Colorado ${ }^{2}$ Henry Ford Health System, Detroit, Michigan; ${ }^{3}$ Flagler College Department of Communications, St Augustine, Florida; ${ }^{4}$ Kaiser Permanente Center for Health Research, Honolulu, Hawaii; ${ }^{5}$ Kaiser Permanente Center for Health Research, Portland, Oregon; ${ }^{6}$ University of Colorado Cancer Center, Denver, Colorado.

Judy Mouchawar, MD, MSPH, Kaiser Permanente Colorado, Dept. Prev Med, 580 Mohawk Drive, Boulder, CO 80302.

Received: November 1, 2004.

Accepted: January 3, 2005.
}

DOI: 10.1097/01.GIM.0000156526.16967.7A message explaining hereditary risk for breast and ovarian cancers and options for obtaining more information (Table 1).

The DTC-ad began in mid-September 2002 and continued through mid-February 2003. ${ }^{4}$ The intensive 5 -month advertising campaign used television, radio, and print media, and was projected to reach more than $90 \%$ of the targeted population an average of 16.5 times each. The advertising plan targeted women aged 25 to 54 by placing advertisements in programs and magazines aimed at this demographic, and subtargeted women with a family history of breast cancer by using ad content that presented four middle-aged female actors stating that breast cancer ran in their families and they had sought testing.

The effect of the DTC-ad on cancer genetic services was uncertain as such has not been previously marketed and the literature on marketing of genetic testing outside of cancer prediction provided little insight. However, within the pharmaceutical literature there is a clear indication that marketing product directly to the consumer leads to increased sales. ${ }^{5-10}$ Thus, we anticipated an increase in demand for counseling and testing services due to DTC-ad of cancer genetic testing, but were uncertain to what degree.

While hypothesizing an increase in demand for testing, we did not assume that overuse of testing would occur. Although 
Table 1

KPCO's services in anticipation and during Myriad Genetic Laboratories, Inc.'s direct-to-consumer advertising campaign for BRACAnalysis

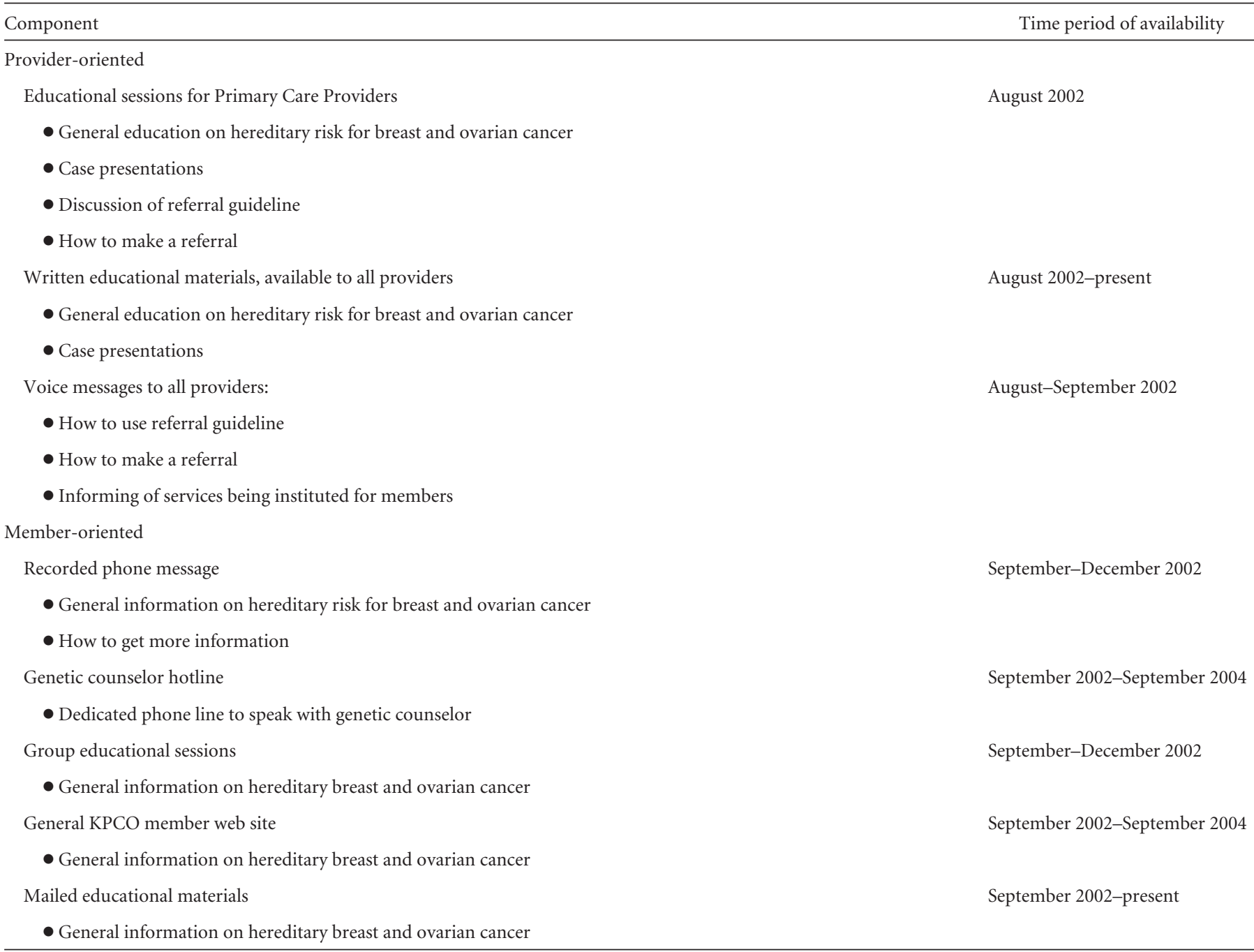

this contradicts others, cancer genetic counseling and testing services are a covered benefit for KPCO members, and testing criteria exist to determine benefit coverage. ${ }^{11-13}$ Thus, we did not anticipate that testing would be overused. We did hypothesize that such advertising could lead to demand for cancer genetic counseling or clinical cancer genetic services that could quickly overwhelm clinic capacity, as have others. ${ }^{12,13}$

The primary objective of this study was to describe the impact of the first ever marketing campaign for BRACAnalysis within a large, nonprofit Managed Care Organization (MCO) in Denver, Colorado, on genetic counseling referrals and level of mutation probability among such referrals as well as mutation probability of those undergoing testing.

\section{METHODS}

\section{Study sites}

Kaiser Permanente Colorado (KPCO) is a nonprofit, closed panel MCO serving nearly 370,000 members in the Denver
Metropolitan Area. Clinical practice referral guidelines for cancer genetic services have been in place at KPCO since 1997 and include a requirement of pretest genetic counseling. Referral for such services must be made by a health care provider, as there is no self-referral system in place. KPCO has established testing criteria for benefit coverage of $B R C A 1 / 2$ sequencing. In the year prior and throughout the DTC-ad, there were two genetic counselors and one oncologist who saw all KPCO patients for cancer genetic counseling and testing.

That the DTC-ad occurred in only two large metropolitan areas provided opportunity for a natural experiment. By comparing the experience of KPCO to an MCO where the population was not exposed, we aimed to more precisely assess the impact of the campaign against any time trends that also may have been occurring. Henry Ford Health System (HFHS) is the largest MCO in Southeastern Michigan, and served as a comparison community.

A portion of the HFHS membership is similar to KPCO in terms of being a closed-panel MCO. Only that portion of the 
HFHS membership was used for this study so as to maintain similarity of services whereby members are only financially responsible for copays. HFHS is similar to KPCO in terms of size and cancer genetic testing market share (Table 2). We acknowledge that the HFHS membership is ethnically different from KPCO. However, we expect any change in utilization of and access to cancer genetic services among insured women to be independent of race/ethnicity, and the estimate of the relative differences in referrals across time and site to be accurate. ${ }^{14,15}$ Thus, utilizing the closed-panel portion of HFHS as a comparison community allowed description of the proportion of the KPCO referral increase due to the DTC-ad separated out from temporal or secular changes in demand derived from other sources.

\section{Statistical analysis}

The study was approved by the KPCO and HFHS Institutional Review Boards (IRBs). Both KPCO and HFHS maintain an electronic cancer genetic database of the referral, counseling, and testing processes for tracking and improvement purposes, which we used to assess the impact of the DTC-ad on service demand.

The difference in number of referrals approved for breast/ ovarian cancer genetic services at KPCO from July 2001 to March 2002 (pre-DTC-advertising or PDA) and July 2002 to March 2003 (DTC-advertising or DA) was compared to the

Table 2

Demographics of Henry Ford Health Systems (HFHS) and Kaiser Permanente Colorado (KPCO) membership, December 2002

\begin{tabular}{|c|c|c|}
\hline & KPCO & HFHS \\
\hline & Number (\%) & Number $(\%)$ \\
\hline Plan size & $367,795(100)$ & $290,205(100)$ \\
\hline \multicolumn{3}{|l|}{ Sex } \\
\hline \multicolumn{3}{|l|}{ Female } \\
\hline $25-54$ y & $83,931(23)$ & $75,021(26)$ \\
\hline $55-84$ y & $51,087(14)$ & $33,384(12)$ \\
\hline $85 \mathrm{y}$ and older & $3,301(1)$ & $1,539(1)$ \\
\hline \multicolumn{3}{|l|}{ Male } \\
\hline $25-54$ y & $76,207(21)$ & $56,508(19.5)$ \\
\hline $55-84$ y & $42,186(12)$ & $28,571(9.8)$ \\
\hline $85 \mathrm{y}$ and older & $1,508(0.4)$ & $884(0.3)$ \\
\hline \multicolumn{3}{|l|}{ Race/Ethnicity } \\
\hline Non-Hispanic White & $275,846(75)$ & $166,347(57.3)$ \\
\hline Hispanic & $66,203(18)$ & $1,490(0.5)$ \\
\hline African-American & $14,712(4)$ & $96,634(33.3)$ \\
\hline Other & $11,034(3)$ & $25,734(8.9)$ \\
\hline \multicolumn{3}{|l|}{ Market Share $^{a}$} \\
\hline BRCAnalysis & $15.8 \%$ & $16.5 \%$ \\
\hline
\end{tabular}

difference at HFHS during the same two time periods. Differences were tested for statistical significance using Chi-square of proportions testing, with denominators being numbers enrolled at each of the health plans in December of the respective year.

We also assessed differences in referrals by pretest probability for a $B R C A 1 / 2$ mutation between the two time periods. To do this, we identified personal and family cancer histories and Jewish ancestry from the referral databases at KPCO and HFHS, and used this information in the Myriad Mutation Prevalence Tables (MPT) to obtain a risk assessment for testing positive. ${ }^{16}$ Although there are other computer-based mutation probability assessment models, the MPT is the only tool based on actual test results and best fit the level of information available.

Using the MPT, we created a high probability category for patients that equated to a $10 \%$ or greater chance of testing positive for a BRCA1/2 mutation. To assure that the MPT gave appropriate probabilities, at KPCO, we randomly pulled 10 self-reported family history information (from the precounseling questionnaire) from each month of the DA and compared this to the initial referral (from which we calculated mutation probability using the MPT). We found that the referral generally indicates the same or higher mutation probability level than the self-reported survey information. Further, we utilized BRCAPRO, a widely utilized mutation assessment tool, across 20 various family histories and found that the MPT consistently gave higher mutation probability estimates. ${ }^{17-18}$ Thus, we were confident that using the MPT would not underestimate mutation probability.

Regardless of the tool used, because the probability criteria were kept the same for the two time periods and for the two MCOs, the data reported are an accurate estimate of relative differences across time and site. Statistically, characteristics of referrals in the PDA and the DA at KPCO were compared using Chi-square of proportions or Fisher exact tests. The denominator for comparisons between the two time periods were the respective numbers of referrals generated during that time period. All analyses were done using SAS Version 8.02 (SAS Institute Inc, Cary, NC).

\section{RESULTS}

During the first 3 months of the active DTC-ad campaign, there was an average of 120 member requests for information per day to one of the member-oriented services outlined in Table 1 (data not shown). Requests then tapered and declined to an average of 10 requests per day by the end of the DA.

Over the entire DA, referrals at KPCO increased more than $240 \%$ over PDA $(P<0.001)$ (Table 3$)$. Although the numbers of referrals during the DA were higher than PDA for every month, referral increase was highest toward the beginning of the DTC-ad and during October, Breast Cancer Awareness month, with waning over the holiday months and as the campaign ended (Fig. 1). In comparison, referrals at HFHS did not increase over the two time periods $(P=0.94)$. 
Table 3

Numbers and BRCA1/2 pretest mutation probability of referrals for hereditary breast and ovarian cancer during July 2001-March 2002 (Period 1) and July 2002March 2003 (Period 2) at Kaiser Permanente Colorado (KPCO) and Henry Ford Health Systems (HFHS)

\begin{tabular}{|c|c|c|c|c|c|c|}
\hline & \multicolumn{3}{|c|}{ KPCO Number (percent) } & \multicolumn{3}{|c|}{ HFHS Number (percent) } \\
\hline & Period 1 & Period 2 & $P$ value & Period 1 & Period 2 & $P$ value $^{a}$ \\
\hline Total number referrals per average membership ${ }^{b}$ & $144(100)$ & $499(100)$ & $<0.001$ & $53(100)$ & $52(100)$ & 0.94 \\
\hline High mutation probability $^{c}$ & $100(69)$ & $238(48)$ & $<0.001$ & $35(66)$ & $33(63)$ & 0.78 \\
\hline
\end{tabular}

${ }^{a}$ All $P$ values obtained using chi-square.

${ }^{b}$ The denominator for total number of referrals is average membership during the time period.

${ }^{c}$ High mutation probability = probability of finding a BRCA1/2 mutation at least $10 \%$, as per Myriad Mutation Prevalence Tables.

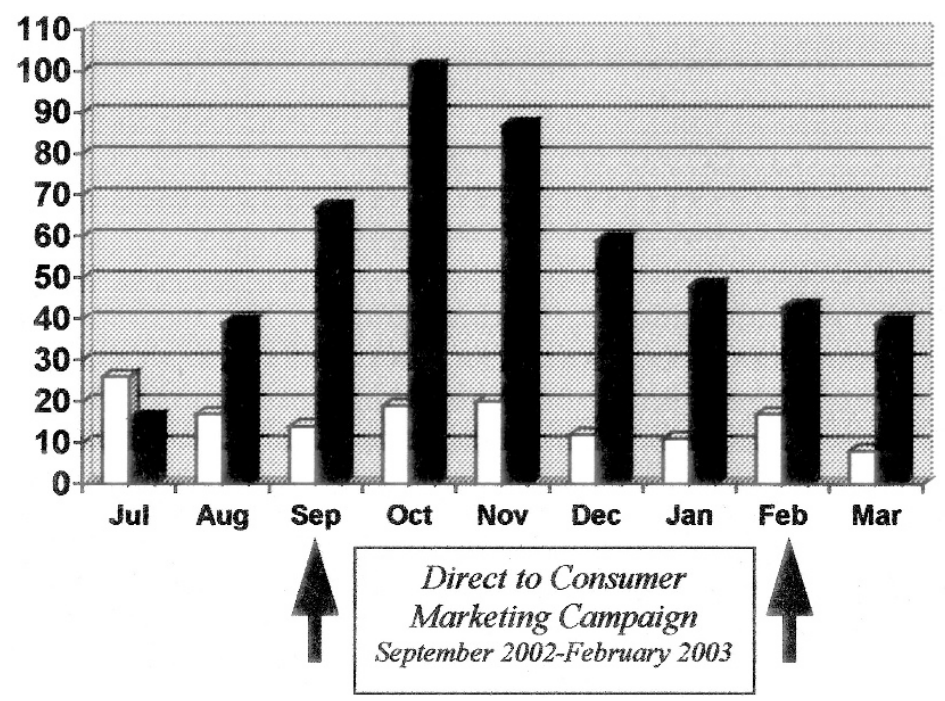

\section{口Period 1 \\ Period 2}

Fig. 1. Numbers of referrals for breast and ovarian cancer genetic services by month at Kaiser Permanente Colorado for July 2001 to March 2002 (Period 1) and July 2002 to March 2003 (Period 2).

Although the number of referred women at KPCO with a high pretest probability for a $B R C A 1 / 2$ mutation increased during the DA, the proportion decreased from an average of $69 \%$ in the PDA to $48 \%$ during the DA $(P<0.001)$ (Table 3$)$. The proportion of high mutation probability referrals was lower during the DA for every month of the active DTC-ad (Fig. 2). At HFHS, there was no significant difference in mutation risk among referred women between the two time periods $(P=0.78)$.

Because of the significant differences seen in the KPCO referrals between the two time periods, we further assessed characteristics of those referrals (Table 4). Of the KPCO women referred, the majority were between the ages of 30 and 64 and had been members for at least 5 years. More unaffected women, those without a cancer diagnosis, were referred during the DA. Referrals were predominantly made by physician gynecologists during both the PDA and DA, although it appears that the DTC-ad raised awareness among physicians of specialties outside of primary care and among nonphysician providers.

While the onslaught of referrals resulted in delayed access, a similar percentage of women referred during the DA actually attended counseling as PDA (Table 3). The percentage of re- ferrals undergoing testing varied between the two time periods, likely reflecting the change in the proportion meeting testing criteria $(P<0.01)$, as there was no increase of testing among women with a low mutation probability during the DA (DA = $3.6 \%$, PDA $=2.5 \%, P=1.0$, data not shown). Similarly, at HFHS, there was no change in testing among women with a low probability for mutation ( $P=0.1$, data not shown).

\section{DISCUSSION}

As this first DTC-ad campaign for BRCA1/2 testing was initiated, publications emerged with concerns of the overall and ethical appropriateness of DTC-ad for genetic testing. ${ }^{12,13,19-21}$ Such concerns included the possibility for disproportionate demand for services and that, with the paucity of experts in cancer genetics, the potential DTC-ad effect of significantly increasing demand for services could strain many aspects of the medical community. Overall, our study showed that there was a highly significant increase in demand for genetic information at KPCO during the DA that was not seen in the HFHS comparison site.

A study conducted concurrently by the Centers for Disease Control on the effects of the marketing campaign on the gen- 


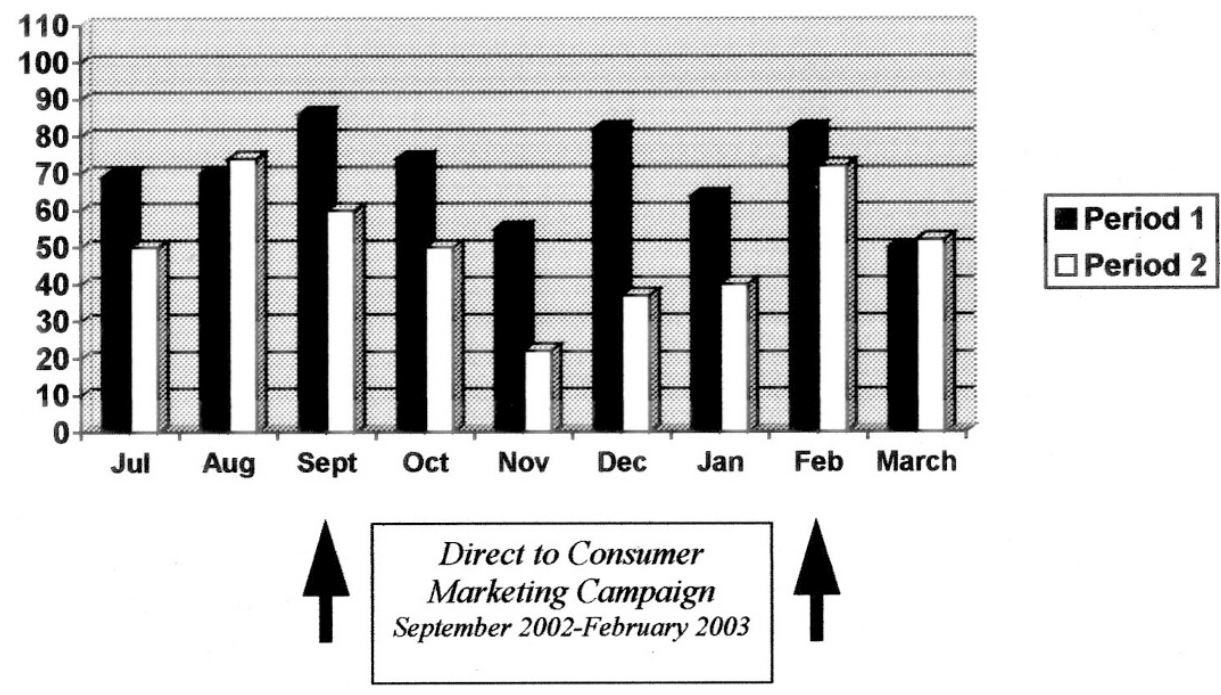

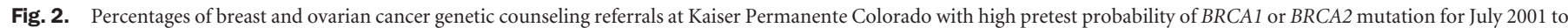
March 2002 (Period 1) and July 2002 to March 2003 (Period 2).

eral population reported that although patient self-report of seeking genetic testing did not appear to be affected by the advertising campaign, physicians' report of more patients making genetic testing inquiries were associated with the ad campaign. ${ }^{22}$ Whereas the physician reports align with our findings, the consumer reports do not. The contradiction has a number of explanations including that self-report measures may not be as reflective of reality as actual referral numbers. Further, report of interest among the general population would be expected to be lower than among a group of women with prepaid access to health care. Members of KPCO may also have been "helped along" in their interest for cancer genetic services by the physician and patient education efforts instituted by KPCO, and/or by informal conversations in workplaces or other settings where women had insurance coverage in common.

In our study, that a significant number of women with lower probability for mutation were referred as a result of the DTC-ad did not surprise us. Previous literature suggests that primary care providers are not well-informed to discuss the issues of cancer genetics with patients. ${ }^{23-26}$ Further, the advertisements were aimed at a vaguely defined "family history" of breast or ovarian cancer. That is, the DTC-ad encouraged women with a family history of breast or ovarian cancer to talk with their health care provider about BRACAnalysis. Without a more specific definition of family history, it is likely that women with a lower probability for mutation would consider themselves to be at a higher probability.

However, this is not to say that these women should not be referred. Indeed, of the KPCO referrals during the DTC-ad, only $8(1.6 \%)$ were denied due to extremely low probability $(<$ $2 \%$ ) of a mutation (data not shown). The majority of the lower risk women referred had some level of risk for which further assessment of mutation probability and/or discussion of risk in a counseling session was warranted. With any future DTC advertising, in the face of broad advertising messages and gaps in provider knowledge, referrals of women with lower mutation probability will likely occur. Thus, the future challenge for providers and organizations will be to provide these services while not hindering counseling and testing services from women with higher mutation probability.

As the advertising campaign started, KPCO was almost immediately faced with this challenge. Although not conducted in rigorous scientific fashion, two services were quickly implemented to handle the access delay of over 6 months. A triaging system was implemented such that women making surgical decisions and those with high mutation risk were seen first. Women at lower risk were mailed multiple letters with explanation of the delay in services and with provision of information including invitation to a number of group educational sessions.

Through communication with all of the other genetic counselors in the Denver Metropolitan Area providing cancer counseling, we found that $<5$ women went outside of KPCO during the DA to receive counseling due to extended wait times. Furthermore, KPCO received no patient or provider complaints to customer service or to cancer genetics throughout the DA. Thus, although lower risk women should be able to receive cancer genetic information, until there is a greater understanding of this area among nongenetic health care providers, a combination of approaches from those directly in cancer genetics may be needed.

Our study data show that there was no increase of testing among women with a $<10 \%$ pretest probability for mutation. We considered that women who responded to a DTC-ad might feel more urgent about their risk and therefore be more demanding of testing during the counseling session, particularly among those with less mutation probability. However, based on personal communication with the two cancer genetic counselors seeing patients for KPCO, this did not occur, and difficult counseling conversations due to low risk women demanding to be tested did not occur. 
Table 4

Characteristics of the referrals for hereditary breast and ovarian cancers during July 2001-March 2002 (Period 1) and July 2002-March 2003 (Period 2) at Kaiser Permanente Colorado

\begin{tabular}{|c|c|c|c|}
\hline & $\begin{array}{c}\text { Period } 1 \\
\text { Number }(\%)\end{array}$ & $\begin{array}{c}\text { Period } 2 \\
\text { Number }(\%)\end{array}$ & $P$ value $^{a}$ \\
\hline Age & & & 0.27 \\
\hline Less than 30 & $19(13)$ & $58(12)$ & \\
\hline $30-39$ & $37(26)$ & $128(26)$ & \\
\hline $40-49$ & $49(34)$ & $137(27)$ & \\
\hline $50-64$ & $33(23)$ & $144(29)$ & \\
\hline $65-69$ & $5(3)$ & $15(3)$ & \\
\hline 70 and older & $1(1)$ & $17(3)$ & \\
\hline Length of enrollment prior to referral & & & 0.48 \\
\hline$<3$ years & $52(36)$ & $156(31)$ & \\
\hline 3 to $<5$ years & $18(13)$ & $58(12)$ & \\
\hline 5 to $<10$ years & $32(22)$ & $105(21)$ & \\
\hline $10+$ years & $42(29)$ & $180(36)$ & \\
\hline Jewish Ancestry & & & 1.00 \\
\hline & $3(2)$ & $10(2)$ & \\
\hline Personal history breast cancer $<50$ & & & $<0.01$ \\
\hline & $20(14)$ & $29(6)$ & \\
\hline Personal history breast cancer $>50$ & & & 0.19 \\
\hline & $3(2)$ & $4(1)$ & \\
\hline Personal history ovarian cancer & & & $<0.01$ \\
\hline & $4(3)$ & $0(0)$ & \\
\hline Specialty of referring provider & & & $<0.01$ \\
\hline Gynecologist & $74(51)$ & $281(56)$ & \\
\hline Internal Medicine & $30(21)$ & $75(15)$ & \\
\hline Family Practice & $12(8)$ & $57(11)$ & \\
\hline Surgery & $11(8)$ & $13(3)$ & \\
\hline Oncology & $17(12)$ & $23(5)$ & \\
\hline Other & 0 & $50(10)$ & \\
\hline Discipline of referring provider & & & $<0.01$ \\
\hline $\mathrm{MD}$ or $\mathrm{DO}$ & $117(81)$ & $272(55)$ & \\
\hline NP or PA & $27(19)$ & $179(36)$ & \\
\hline Other & 0 & $48(10)$ & \\
\hline \multirow{2}{*}{$\begin{array}{l}\text { Women utilizing counseling within } \\
18 \text { months of the referral date }\end{array}$} & & & 0.29 \\
\hline & $43(30)$ & $127(25)$ & \\
\hline \multirow{2}{*}{$\begin{array}{l}\text { Women undergoing BRACAnalysis } \\
\text { within } 18 \text { months of referral }\end{array}$} & & & $<0.01$ \\
\hline & $23(16)$ & $28(6)$ & \\
\hline
\end{tabular}

${ }^{a}$ All $P$ values obtained using Chi-square or Fisher's Exact Test.

Through communication with others in the Denver Metropolitan Area providing cancer genetic services, we found that no KPCO member chose to go outside of KPCO to be tested during the DA. Although a limited number of KPCO women were counseled out-of-plan during the DA, none were tested. Thus, although referrals for counseling and testing increased greatly as a result of the DTC-ad, no patient went outside of KPCO specifically to receive testing, and the women not meeting testing criteria (whether they expressed seeing the DTC-ad or not) were not demanding of testing services.

Although we doubt that the overwhelming increase in referrals at KPCO during the DA was due to any other outside factor, particularly because there was no increase at HFHS, to ensure the validity of our comparison findings, we communicated with Myriad representatives about testing across the nation. There was no significant unexpected change in testing volume between the two time periods in Kansas City, St Louis, or Detroit, cities that had been designated as control cities before initiating the DTC-ad.

It is possible that counseling demand and uptake as well as the patient information needs could be different in other organizations, in other geographic regions, community practices, or for practices where counseling and testing are not covered benefits. Practices without referral criteria or testing criteria will likely have a difficult time consistently triaging patients during a campaign and thus could have different results from DTC-ad than the experience reported here. However, MCOs are unique in being able to identify patients who are referred for services. It is because of the KPCO and HFHS electronic tracking databases that we are able to provide valuable information on the impact of the DTC-ad on referrals in a timely fashion to other organizations before a DTC-ad campaign begins in their area.

\section{CONCLUSION}

If future direct-to-consumer advertising is conducted, geneticists in particular can expect not only a significant increase in referrals during advertising periods, but also an increase in women with lower mutation probabilities being referred. All specialties can expect an increase in patient request for information and referral request. As such, providers and payers need to think now about the delivery of genetic services and genetic information for persons of all risk levels.

\section{ACKNOWLEDGMENTS}

The authors wish to acknowledge the funding contributions of the Kaiser Permanente Colorado Clinical Research Unit and the Henry Ford Health Systems Josephine F. Ford Endowed Chair in Cancer Genetics.

\section{References}

1. Miki Y, SwensenJ, Shattuck-Eidens D et al. A strong candidate for the breast and ovarian cancer susceptibility gene BRCA1. Science 1994;266:120-122.

2. Wooster R, Neuhausen SL, Mangion J et al. Localization of a breast cancer susceptibility gene, BRCA2, to chromosome 13q12-13. Science 1994;285:2088-2090.

3. Jenks S. BRCA1 patent dispute resolved; NIEHS included. J Natl Cancer Inst 1995; $87: 412-413$.

4. Myriad Genetics, Inc. September 12, 2002. First Ever Campaign for Cancer Predictive Test To Market BRACAnalysis in Denver and Atlanta. Available at: http:// www.corporate-ir.net/ireye/ 
ir_site.zhtml?ticker $=$ mygn\&script $=413 \&$ layout $=9 \&$ item_id $=333030 . \quad$ Accessed December 27, 2004.

5. Holmer AF. Direct-to-consumer prescription drug advertising builds bridges between patients and physicians. JAMA 1999;281:380-382.

6. Kravitz RL. Direct-to-consumer advertising of prescription drugs: implications for the patient-physician relationship. JAMA 2000;284:2244.

7. Tanne JH. Direct to consumer drug advertising is billion dollar business in US. BMJ 1999;319:805.

8. Hollon MF. Direct-to-consumer marketing of prescription drugs: creating consumer demand. JAMA 1999;281:382-384.

9. Wolfe SM. Direct-to-Consumer advertising-education or emotion promotion? $N$ Engl J Med 2002;346:18.

10. Rosenthal MB, Berndt ER, Donohue JM, Frank RG, Epstein AM. Promotion of prescription drugs to consumers. N Engl J Med 2002;346:498-505.

11. Mouchawar J, Goins K, Somkin C, Puleo E, Hensley-Alford S, Geiger A et al. Guidelines for breast and ovarian cancer genetic counseling referral: adoption and implementation in HMOs. Genet Med 2003;5:444-450.

12. Gray S, Olopade O. Direct-to-consumer marketing of genetic tests for cancer: buyer beware. J Clin Oncol 2003;21:3191-3193.

13. Gollust SE, Hull SC, Wilfond BS. Limitations of direct-to-consumer advertising for clinical genetic testing. JAMA 2002;288:1762-1767.

14. Weinick RM, Zuvekas SH, Cohen JW “ Racial and Ethinic Differences in Access to and Use of Health Care Services, 1977 to 1996.” Medical Care Research and Review, Vol 20; 2000:36-54.

15. Cohen JW, Monheit AC, Beauregard KM, Cohen SB, et al. "The Medical Expenditure Panel Survey: A National Health Information Resource.” Inquiry 1996;33:373389.
16. Myriad. 2004. Mutation Prevalence Tables. Available at: http://www.myriadtests.com/provider/mutprevo.htm. Accessed December 27, 2004.

17. Domchek SM, Eisen A, Calzone K, Stopfer J, Blackwood A, Weber BL. Application of breast cancer risk prediction models in clinical practice. J Clin Oncol 2003;21:593601.

18. BRCAPRO. Available at: http://astor.som.jhmi.edu/brcapro/. Accessed December 27, 2004.

19. Graham J. Gene-test makers woo consumers: Pitches exploit fear of cancer, critics complain. Chicago Tribune Sep 8, 2002:12.

20. Austin M. Marketing of cancer test exploits fears, critics say. Denver Post August 30, 2002.

21. Flam F. Gene tests' accuracy reassessed; disease-risk figure may be overstated. Philadelphia Inquirer September 17, 2002:A01.

22. Centers for Disease Control and Prevention. Genetic testing for breast and ovarian cancer susceptibility: evaluating direct-to-consumer marketing: Atlanta, Denver, Raleigh-Durham, and Seattle, 2003. MMWR 2004;53:603-606.

23. Mouchawar J, Klein C, Mullineaux L. Colorado physicians' hereditary breast cancer knowledge and practice behavior. J Cancer Educ 2001;16:33-37.

24. Wideroff L, Freedman AN, Olson L, Klabunde CN, Davis W, Srinath KP, et al Physician use of genetic testing for cancer susceptibility: results of a national survey. Cancer Epidemiol Biomarkers Prev 2003;12:295-303.

25. Acton RT, Burst NM, Casebeer L, Ferguson SM, Greene P, Laird BL, Leviton L. Knowledge, attitude, and behaviors of Alabama's primary care physicians regarding cancer genetics. Acad Med 2000;75:850-852.

26. Escher M, Sappino AP. Primary care physicians' knowledge and attitudes towards genetic testing for breast-ovarian cancer predisposition. Ann Oncol 2000;11:11311135 . 